\section{In Reply: Issues of Outcome in Gastric Cancer}

\section{TO THE EDITORS:}

In the letter by Chalkiadakis and Ziogas, the authors provide a brief but exhaustive update of the worldwide fight against gastric cancer. ${ }^{1}$ As the authors point out, this battle has been largely successful in Japan, but not in the Western world. As Chalkiadakis and Ziogas underline, our work brings further support to the notion that the excellent short-term results achieved by Japanese surgeons can be largely reproduced in the Western world. ${ }^{2}$

In our article, we also sought to propose indexes of surgical quality for gastric cancer surgery, which are presently lacking. In systematic reviews, papers dealing with gastric cancer surgery are mainly evaluated on the basis of study design, while surgical quality is completely overlooked.

We share the authors' hope that early tumor detection and personal genomics will improve prognosis of advanced gastric cancer in the Western world, but much effort is still necessary to translate these hopes into everyday clinical practice. In our series, the $\mathrm{T}$ stage of patients undergoing gastrectomy did not vary significantly during the observation period (1988-2002).

The authors conclude that "high-quality evidence suggests that more extensive surgery such D2 lymphadenectomy plus para-aortic lymph nodal dissection does not provide any survival benefit." Indeed, the Japan Clinical Oncology Group (JCOG) trial demonstrated that para-aortic node dissection does not improve survival in gastric cancer patients without macroscopic metastases to nodes of the 16th station. $^{3}$ Nevertheless, it cannot be excluded that selected categories of patients could benefit from para-aortic nodal excision, such as patients with metastases to the latter station. In the JCOG trial, 5-year survival in patients with metastases to para-aortic nodes was $18 \%$. At present, the following approach is emerging within the Italian Research Group for Gastric Cancer: first, patients at high risk for para-aortic nodal invasion should be identified by considering site, histology, depth of tumor invasion, and, in borderline situations, status of perigastric lymph nodes; then, the indication for D3 lymphadenectomy should be restricted to these patients, who in our series represent $<50 \%$ of all patients with advanced gastric cancer. ${ }^{4}$

Giovanni De Manzoni, MD $^{1}$, Giuseppe Verlato, MD, $\mathrm{PhD}^{2}$, Alberto Marchet, $\mathrm{MD}^{3}$, and Franco Roviello, $\mathrm{MD}^{4}$ ${ }^{1}$ First Division of General Surgery, Borgo Trento Hospital, Verona, Italy;

${ }^{2}$ Epidemiology and Medical Statistics, University of Verona, Verona, Italy;

${ }^{3}$ Second Division of Surgery, University of Padua, Padua, Italy;

${ }^{4}$ Division of Surgical Oncology, University of Siena, Siena, Italy

e-mail: giuseppe.verlato@univr.it

Published Online: 29 May 2009

(C) Society of Surgical Oncology 2009

\section{REFERENCES}

1. Chalkiadakis GE, Ziogas D. The decisive role of surgeon's experience in short-term and long-term oncological outcomes and quality of life in gastric cancer. Ann Surg Oncol. 2009;16. DOI:10.1245/s10434-009-0518-1.

2. Verlato G, Roviello F, Marchet A, et al. Indexes of surgical quality in gastric cancer surgery: experience of an Italian network. Ann Surg Oncol. 2009;16:594-602.

3. Sasako M, Sano T, Yamamoto $S$, et al, for the Japan Clinical Oncology Group. D2 lymphadenectomy alone or with para-aortic nodal dissection for gastric cancer. N Engl J Med. 2008;359:45362.

4. de Manzoni G, Verlato G, Roviello F, et al. Site and perigastric nodal status are the most important predictors of para-aortic nodal involvement (abstract). Paper presented at: 8th international gastric cancer congress. 